\title{
La naissance de la SFRP
}

\author{
D. BLANC ${ }^{1}$ et les membres du club « histoire »
}

(Manuscrit reçu le 25 novembre 2002, accepté le 6 janvier 2003)

\begin{abstract}
RÉSUMÉ Voici la naissance d'une nouvelle rubrique de notre revue : « un peu d'histoire». Puisqu'il est question de naissance, il nous a paru logique de traiter celle de la SFRP. On montre comment la création de l'International Radiation Protection Association (IRPA) a pour conséquence le regroupement des radioprotectionnistes français, qui se partageaient entre deux associations, l'une dominée par des ingénieurs, l'autre par des médecins. Nous évoruons les épisodes successifs qui conduiront au dépôt en préfecture des statuts de la SFRP, le 18 février 1965 ; les archives ne précisent pas à quelle heure.
\end{abstract}

ABSTRACT Birth of the French Society for Radiation Protection, SFRP.

Here is the birth of a new category of our journal: "a little history". Since it is a question of birth, it appeared logical to us to treat that of the SFRP. We show how the creation of the International Radiation Protection Association (IRPA) has as a consequence the regrouping of the French radioprotectionnistes, shared between two associations, one dominated by engineers, the other by physicians. We evoke the successive episodes that lead to the depositing in the prefecture of the statutes of the SFRP on February 18, 1965; the archives do not specify at what time.

\section{La préhistoire en Europe}

Nous entendons par-là la période allant jusqu'à 1964. Différents pays européens disposaient alors d'une ou plusieurs sociétés de radioprotection (deux en France) mais il n'y avait pas de regroupement international. Il existait, parmi les professionnels de la radioprotection allemands, belges, français et néerlandais, des européens presque chevronnés, une certaine volonté de créer une société européenne de radioprotection, l'initiative étant allemande. Des efforts ont été faits pour la constituer, mais tout sera abandonné, suite à la création de l'IRPA qui, comme on le verra après, est à l'origine de la SFRP (Lochard, 2000). Le souci de constituer une société européenne est une volonté de créer un pôle de professionnels en Europe face au pôle américain déjà bien puissant et organisé (Hébert, 2000).

\footnotetext{
I I place de l'église, 31570 Lanta, France.
} 


\section{L'événement déterminant : la création de l'IRPA}

Depuis leurs premières années d'activité dans le domaine nucléaire (le projet Manhattan), les États-Unis possèdent une société de radioprotection, la Health Physics Society (HPS). En 1963-64, sous la présidence de W.T. Ham, cette société prend l'initiative de promouvoir une société internationale, dont le nom serait l'«International Radiation Protection Association (IRPA)», et dans laquelle viendrait se fondre la HPS, qui en formerait la composante la plus importante.

Le conseil de la HPS charge K.Z. Morgan de sonder l'opinion internationale et de prendre contact avec des personnalités très connues en radioprotection, dans le monde entier. Ces contacts ont été fructueux, puisque la HPS constitue un comité ad hoc formé de 46 personnes venant de 25 pays (Maushart, 2000).

Ce comité nomme un groupe de travail de 11 personnes, afin de définir les principes de fonctionnement, la finalité, les objectifs précis de cette nouvelle société. La première réunion de ce groupe a lieu à Londres, le $1^{\text {er }}$ février 1964. À partir de là, l'émergence de l'IRPA est rapide. Ces onze «pères fondateurs », tous présents le $1^{\text {er }}$ février 1964, sont :

A. Benco (Italie), P. Courvoisier (Suisse), P. Bonet-Maury, F. Duhamel, H. Jammet (France), W.G. Marley, B.A.J. Lister (UK), S. Halter (Belgique), R. Maushart (Allemagne), W.T. Ham, K.Z. Morgan (E.U.).

Ce cercle initial est élargi pour former un conseil exécutif provisoire, qui se réunit le 12 juin 1964 à Gatlinburg (ÉU). À cette réunion le nom IRPA est approuvé, et l'on commence à jeter les bases des statuts de cette association, sous l'arbitrage de J.C. Hart. Trois jours plus tard, le conseil présente, à Cincinnati (ÉU), ses propositions au bureau de la HPS, qui les approuve, et approuve la composition du conseil. Il est enfin décidé de tenir l'assemblée générale constitutive à la fin de 1964.

Cette assemblée va se tenir à Paris, sur l'initiative de la section française de la HPS.

L'objectif de l'IRPA est de rassembler un nombre aussi grand que possible de sociétés nationales, avec la règle de n'admettre qu'une seule société par État, ce qui posera problème en France où deux sociétés cohabitaient. Les statuts indiquent que les membres d'une société nationale associée deviennent également membres de l'IRPA. Pour les élections internes à l'IRPA, chaque société dispose d'un nombre de voix proportionnel au nombre de ses adhérents (une voix pour 100 adhérents). 


\section{La préhistoire en France}

Pourquoi y avait-il trois français dans le groupe fondateur de l'IRPA ? Comment avaient-ils été « choisis »? Ce qui suit apporte la réponse.

Avant 1964, il existe, en France, deux sociétés de radioprotection.

La plus importante est la société des ingénieurs en radioprotection (SIRP), qui est aussi la section française de la Health Physics Society. Elle est ainsi affiliée à la société qui crée l'IRPA. Pourquoi une telle affiliation ? Cela peut s'expliquer par le fait que l'HPS au-delà de ces membres (3000 environ) comporte aussi des étrangers, de 47 pays, et est riche d'un grand nombre de compétences, ce qui est des plus précieux pour les « affiliés » comme la SIRP qui regroupe des spécialistes des domaines scientifiques et techniques comme F. Duhamel, polytechnicien, qui dirige le service central de radioprotection et de génie radioactif (SCRGR) du CEA.

La seconde est la société de radioprotection (SRP), animée par H. Jammet, docteur en médecine. Elle est numériquement moins importante, mais son animateur est un fin manouvrier ; l'avenir le démontrera. Elle regroupe beaucoup de médecins, en particulier du CEA. H. Jammet dirige le service de protection radiologique (SPR) du CEA.

On voit ainsi apparaître, de manière encore floue, la future compétition entre « ingénieurs » et « médecins».

À partir de juin 1964 ont lieu des pourparlers entre les deux sociétés, dans des conditions favorables, car, par chance, le président de la SIRP est N. ChassendeBaroz, pharmacien général de la défense nationale, homme d'une très grande ouverture d'esprit ${ }^{2}$. Il est l'homme de la situation pour faire aboutir les discussions entre les deux «composantes ». Son caractère affable et tolérant, sa patience, son altruisme, en font le créateur idéal d'une fusion heureuse. Premier président de la SFRP, il sera nommé, ultérieurement, «président fondateur » et «membre d'honneur ». Dans ces négociations, il est secondé par le professeur J. Chanteur, du SCPRI, médecin universitaire. «Furent alors péniblement élaborés les statuts qui devaient permettre la fusion des deux sociétés précédentes » (Duhamel, 1970).

\footnotetext{
2 Il subsiste quelques doutes sur les rôles respectifs de N. Chassende Baroz er de P. Bonet-Maury au sein de lá SIRP. Nous pensons que le premier, président de la SIRP, devient président fondateur de la SFRP. P. Bonet-Maury le remplace à la tête de la SIRP, pour peu de temps, puisqu'il succède à N. Chassende Baroz, et devient président élu de la SFRP.
} 


\section{SIRP : la réunion du 28 septembre 1964}

Nous ne possédons aucun documents portant sur les discussions préliminaires ; elles ont été relativement rapides : de juin à novembre 1964.

Lors de sa réunion du 28 septembre 1964, le conseil d'administration de la SIRP adopte le principe d'une fusion entre les deux sociétés. La composition du conseil est la suivante : MM. Bazire (LCA), Bonet-Maury (Orsay), ChassendeBaroz (armées, président), Duhamel (CEA), François (CEA), Gras (CEA), Joffre (CEA), Pellerin (professeur, directeur du SCPRI), Pradel (CEA). L'unanimité est de façade ; en effet, M. Pellerin « exprime des doutes sur la nécessité d'une fusion immédiate, et insiste particulièrement sur les garanties qui doivent être offertes, de part et d'autre, pour que les statuts de la nouvelle société ne puissent être modifiés unilatéralement »(Collectif, 1964).

Le conseil note que c'est lorsque l'HPS deviendra « section américaine de l'IRPA » que la question de la dissolution de la SIRP pourra être posée à l'assemblée générale de la SIRP, à l'initiative de son conseil d'administration (Collectif, 1964).

Le conseil désigne quatorze membres fondateurs de la nouvelle société, appelés à siéger dans son conseil provisoire. Il s'agit de MM. Allisy (CNAM), Bazire, Chanteur, Bonet-Maury, Chassende-Baroz, Deforge, Delpla (EDF), Duhamel, François, Gras, Joffre, Pellerin, Pomarola (CEA), Pradel.

\section{Vers la création de la SFRP}

De son côté, la SRP tient son assemblée générale le 9 novembre 1964. Elle décide de "joindre ses efforts à ceux de nos collègues de la Health Physics Society pour que cette association internationale puisse réunir les spécialistes du monde entier » (Bonet-Maury, 1966). Nous ne possédons aucune archive de la SRP. On peut penser que H. Jammet en était alors le président, mais nous n'avons aucune information sur la composition du conseil d'administration.

N. Chassende-Baroz est pressenti pour conduire la SFRP sur les fonds baptismaux ${ }^{2}$. Une ébauche de statuts est créée ainsi qu'un comité provisoire appelé « comité d'administration pour la fondation de la SFRP », regroupant globalement les conseils (et bureaux) des deux sociétés. On précise que « les sociétés anciennes, SRP et SIRP, gardent, jusqu'à nouvel ordre, toute leur indépendance pour leurs activités propres $\gg$. 
Ce comité «accoucheur » compte 24 membres, dont les 14 désignés par la SIRP, dont les noms viennent d'être donnés plus haut. Cette dissymétrie 14-10 semble venir de ce que plusieurs personnes sont membres des deux sociétés. Il s'agit de MM. Bazire, Bonet-Maury, Chanteur, Chassende-Baroz, Delpla et Duhamel. Le premier président de la SFRP a toutes raisons de se trouver parmi ces 6 personnes, mais $\mathrm{N}$. Chassende-Baroz ne souhaite pas jouer ce rôle. Le futur président doit être «neutre ». C'est donc logiquement Bonet-Maury qui est choisi.

\section{IRPA : la réunion historique de Paris, 30 novembre au 3 décembre 1964}

La SFRP n'a pas encore d'existence légale, mais les français agissent comme si c'était le cas. Rappelons que la puissance invitante est la section française de l'HPS, dont le nouveau président est P. Bonet-Maury ${ }^{2} .45$ délégués, représentant 15 sociétés, adoptent les statuts de l'IRPA et élisent un conseil d'administration provisoire, chargé de gouverner jusqu'à la première assemblée générale, qui se tiendra à l'occasion du premier congrès international de l'IRPA, prévu à Rome en septembre 1966.

Le bureau élu est le suivant :

K.Z. Morgan, président (ÉU) ; P. Caldirola, vice-président (Italie) ; P. BonetMaury, secrétaire général (France) ; P. Courvoisier, trésorier (Suisse) et W.S. Snyder, directeur des publications (ÉU).

Les membres du conseil sont :

S. Halder (association belge); K. Becker (section centre Europe de la Health Physics Society). Becker est allemand; R. Maushart (Société européenne de radioprotection) ${ }^{3}$; F. Duhamel (SFRP); H. Jammet (SFRP); Marko (association canadienne) ; Abee (HPS, États-Unis) ; W.T. Ham (HPS, ÉtatsUnis) ; Feige (section israélienne de l'HPS) ; A. Benco (association italienne) ; Nishiwaki (société japonaise); Spaander (société néerlandaise); Sievert (société nordique); Koren (société nordique) ; Marley (section britannique de l'HPS) et Lister (section britannique de l'HPS).

\section{La naissance officielle de la SFRP}

La réunion de Paris accélère l' «alliance » entre les deux sociétés françaises : on ne peut plus reculer, et les statuts de la SFRP sont déposés en préfecture

\footnotetext{
3 Lors de cefte réunion, Maushart a gardé l'étiquctte de lát société européenne, qui, nous l'avons vu, était simplement en projet. En lait, il est l'un des deux représentants de l'Allemagne fédérale.
} 
le 18 février 1965, sous le régime des associations sans but lucratif (loi de 1901). C'est la date officielle de la naissance de notre société.

La première assemblée générale de la SFRP se tient le 17 mai 1965, sous la présidence du général N. Chassende-Baroz. Elle élit le conseil d'administration, qui, à son tour élit comme président $\mathrm{P}$. Bonet-Maury, pour un an, conformément aux statuts. Pharmacien d'origine, il est maître de recherche au CNRS, et chef du service de radioprotection du campus d'Orsay. Il est aussi expert en judo, et a publié un «Que sais-je ? » sur ce sujet. Il cédera son fauteuil à H. Jammet lors de l'AG du 2 juin 1966.

\section{La disparition des deux sociétés antérieures}

La société de radioprotection (SPR) prononce sa dissolution et le transfert de ses comptes, en faveur de la SFRP, en 1967, sous la présidence de H. Jammet.

L'agonie de la SIRP est beaucoup plus longue. En effet, elle marque la continuation de ses activités en élisant, en 1965, un nouveau bureau, formé de MM. P. Pellerin, président, F. Duhamel, président fondateur, M. Gras, secrétaire et $\mathbf{P}$. Candès (CEA), trésorier.

Le 23 novembre 1965, le président Pellerin écrit aux membres de la SIRP une lettre dont nous extrayons des passages (Pellerin, 1965):

"C'est en grande partie grâce aux efforts poursuivis par notre section française de la Health Physics Society qu'une association internationale, à laquelle chaque membre peut s'affilier à titre individuel, a été créée en accord avec toutes les personnalités et toutes les sociétés représentant la radioprotection. [...] De ce fait, la section française de la Health Physics Society va probablement être menée à cesser ses activités en tant que telles. [...] La question se pose de savoir ce que doit devenir notre propre société, si elle doit purement et simplement être dissoute ou si ses membres estiment qu'elle pourrait encore remplir une mission utile. À cet effet, un bulletin vous est adressé, que je vous demande de me retourner sous huitaine. Je vous adresserai le résultat du dépouillement et convoquerai ensuite l'assemblée générale ».

Nous ne possédons aucun document sur ce qui a suivi. Toujours est-il que la dissolution de la SIRP n'est prononcée que le 14 avril 1970 ; elle est annoncée par F. Duhamel au conseil de la SFRP le 22 avril 1970. Il est alors président de la SFRP. L'avoir de la SIRP est versé à la SFRP (Doc, 1970). 


\section{Conclusion}

Un an après sa création, en 1966, la SFRP compte près de 500 membres, ce qui la place en seconde position derrière la HPS, mais très loin derrière. Elle dispose de 5 voix à l'AG de l'IRPA, à Rome, en septembre 1966.

Concluons sur de belles paroles, en citant le rapport moral du président sortant, $\mathrm{P}$. Bonet-Maury, devant l'AG du 2 juin 1966 (Bonet-Maury, 1966) :

"La Société française de radioprotection est née au moment où se faisait sentir l'impérieuse nécessité de regrouper tous les spécialistes qui, à des titres divers, auvrent pour la protection de l'homme et des biens contre les rayonnements ionisants. Que le besoin de cette union ait été aussi nettement ressenti n'est pas un phénomène propre à notre pays : partout dans le monde se conjuguent les efforts pour favoriser les travaux et échanges d'informations dans le domaine de la radioprotection ou pour familiariser l'opinion avec les activités de cette nouvelle discipline ».

Il n'est pas interdit de rêver... Si l'idée d'une société européenne de radioprotection avait pu se concrétiser plus tôt, les européens auraient constitué une force plus importante à côté de l'HPS, et l'IRPA aurait pu se créer dans des conditions différentes. Depuis, l'Europe s'est faite, mais il n'y a toujours pas de société européenne...

\section{RÉFÉRENCES}

Bonet-Maury P. (1966) Radioprotection 1(1), 107.

Collectif (1964) Réunion du 28 septembre 1964, CR déposé par H. Joffre aux archives de la SFRP.

Doc (1970) Doc. 70/1943, PV du CA du 22 avril 1970, archives de la SFRP.

Duhamel F. (1970) Discours devant l'AG de la SFRP, 5 juin 1970.

Hébert J. (2000) CR réunion du club histoire SFRP, 7 juin 2000 , p. 3.

Lochard J. (2000) CR réunion du club histoire SFRP, 7 juin 2000, p. 3.

Maushart R. (2000) Rapport à l'IRPA, 30 mai 2000.

Pellerin P. (1965) lettre du 23 novembre 1965, archives de la SFRP. 